\title{
A Thin Film Teflon Electret Technology for Microphone Applications
}

\author{
Tseng-Yang Hsu, Wen H. Hsieh and Yu-Chong Tai \\ Department of Electrical Engineering \\ California Institute of Technology \\ Pasadena, CA 91125, USA
}

\author{
Katsushi Furutani \\ Department of Mechanical System Engineering \\ Toyota Technological Institute \\ 12-1, Hisakata 2-chome, Tempaku-ku \\ Nagoya 468, Japan
}

\begin{abstract}
A micromachining compatible Teflon electret technology has been developed. The electret material used is thin film spin-on Teflon. A pulsed electron gun, called the Back-Lighted Thyratron (BLT), is used for charge implantation. A charge compensation method is used to measure the charge density of the electret. The electret has a saturated charge density on the order of $10^{-4} \mathrm{C} / \mathrm{m}^{2}$. A prototype silicon micromachined electret microphone has been fabricated and tested. An open circuit sensitivity of $0.3 \mathrm{mV} / \mathrm{Pa}$ has been achieved for a hybrid microphone package.
\end{abstract}

\section{INTRODUCTION}

An electret is a dielectric that produces a permanent external electric field which results from permanent ordering of molecular dipoles or from stable uncompensated surface or space charge. Electrets have been the subject of study for their charge storage characteristics as well as for their application in a wide variety of devices such as acoustic transducers, electrographic devices, and photocopy machines. To incorporate electret technology into micromachining technology is an attractive idea for the fabrication of small, self-powered devices. We have developed and demonstrated a technology to make thin film electrets using micromachining technology. The use of such a technology has been demonstrated in a micromachined microphone which can produce a signal without the need for external biasing. This reduces the system volume and complexity.

\section{ELECTRET FABRICATION}

The electrets fabricated in our laboratory are made from Du Pont spin-on Teflon ${ }^{3}$ AF 1601S. The Teflon dielectric film is prepared by spinning at $2 \mathrm{krpm}$ and baking at $250^{\circ} \mathrm{C}$. The resulting dielectric film is approximately $1 \mu \mathrm{m}$ thick with surface roughness of less than $2000 \AA$ across the wafer. For time spans longer than usual processing times, the adhesion of the Teflon film to different material surfaces such as silicon, silicon dioxide, silicon nitride, copper, gold, and chrome is satisfactory in the presence of chemicals frequently used in MEMS fabrication, such as water, photoresist developers, acetone, alcohol, HF and BHF. If necessary, the Teflon film can be patterned using oxygen plasma.

To form electrets, we have investigated the new use of the Back-Lighted Thyratron for electron implantation into the thin film Teflon. The BLT [1-2] structure we have designed (Figure 1) consists of two electrode plates with hollow back cathode and hollow back anode. The two electrodes facing each other have a diameter of $75 \mathrm{~mm}$ and a center aperture of 5 $\mathrm{mm}$. The electrodes are separated by a $5 \mathrm{~mm}$ thick insulating plexi-glass plate. The structure is filled with a low pressure gas such as hydrogen or one of the noble gases to a pressure of 50 to 300 mTorr. The device is triggered optically by an ultraviolet light pulse applied to the back of the cathode. The ultraviolet light generated by a flashlamp passes through a quartz window into the back of the cathode. This initiates the formation of an electron beam which is directed towards the thin film Teflon sample.

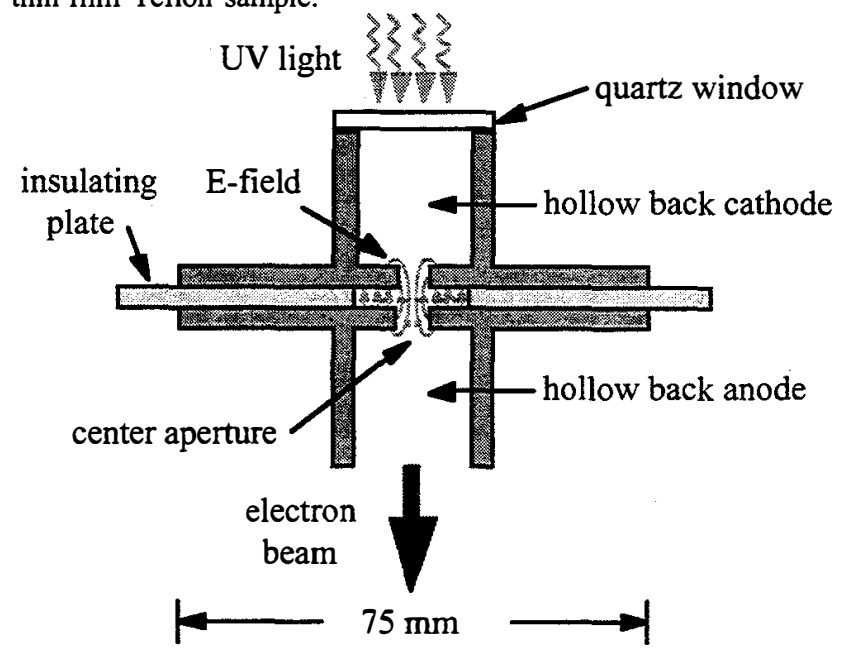

Figure 1. The Back-Lighted Thyratron.

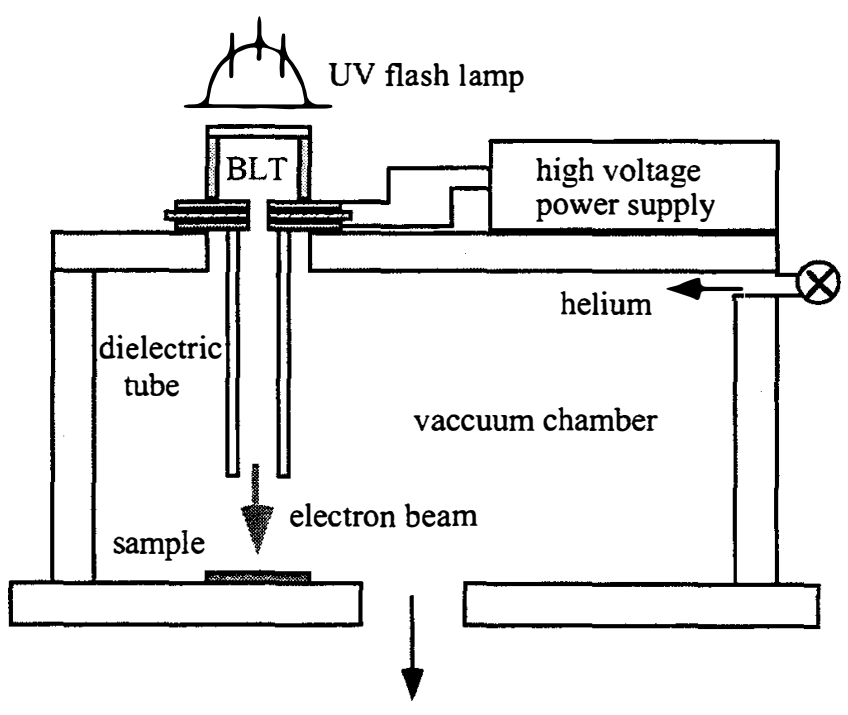

to vacuum pump

Figure 2. The BLT charge implantation system. 
Our in-house BLT is constructed on top of a vacuum chamber with a triggering UV flashlamp at a distance of $2 \mathrm{~cm}$ away from the quartz window as shown in Figure 2. The BLT cathode is biased at a high negative potential for beam acceleration. The electron beam pulse is directed to the sample which is $12 \mathrm{~cm}$ away from the beam exit. With a beam divergent angle of $6^{\circ}$, the beam diameter is $1.75 \mathrm{~cm}$ at the sample surface [2]. Integrating a dielectric tube at the beam exit has the effect of collimating and focusing the electron beam [3]. The bias potential is adjusted according to the desirable range of electrons in the Teflon film. In the case of 1 $\mu \mathrm{m}$ thick Teflon film, the electron beam energy is set at $10 \mathrm{keV}$ which gives an implantation depth of approximately $1 \mu \mathrm{m}[4]$.

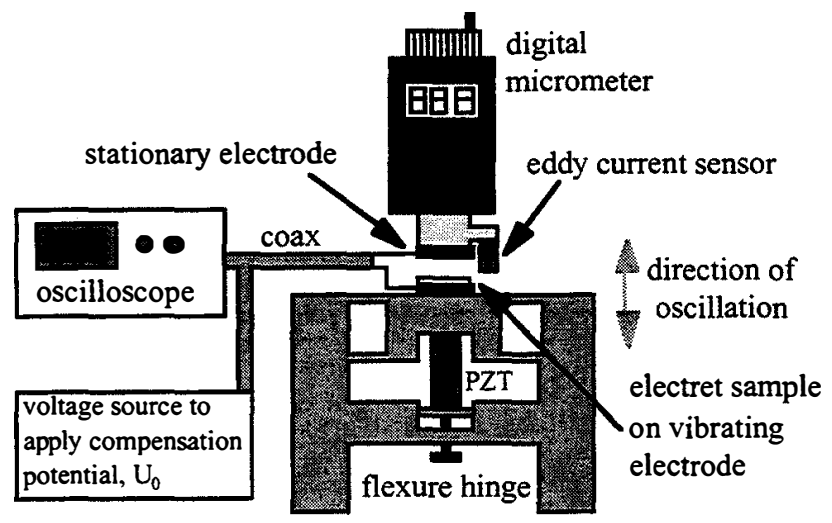

(a)

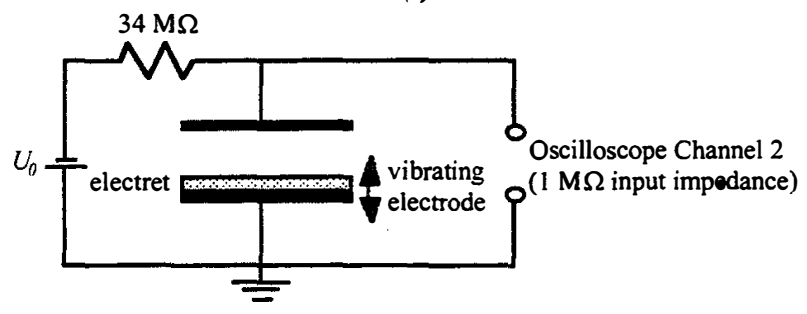

(b)

Figure 3. (a) The setup used to measure the charge density of electret sample. (b) Charge compensation circuit.

\section{ELECTRET CHARACTERISTICS}

To measure the charge density, a setup consisting of a PZT stack and a micrometer controlled stationary electrode was constructed (Figure 3a). To confine displacement in the $z$ direction only, the PZT is integrated into a flexure hinge made of 304 stainless steel and machined by electrical discharge machining (EDM). The movable part of the flexure hinge weighs $30 \mathrm{~g}$ and has a spring constant of $1.53 \times 10^{6} \mathrm{~N} / \mathrm{m}$. The PZT driver deforms $15 \mu \mathrm{m}$ at $100 \mathrm{~V}$ and can be driven by a maximum voltage of $150 \mathrm{~V}$. The linearity of the displacement of the PZT caused by hysteresis is $10 \%$. The PZT is driven by a unit consisting of a periodic source and an amplifier. The amplifier is a class-B push-pull type amplifier specially designed for capacitive loads. An eddy-current sensor is integrated into the micrometer for monitoring and double checking the dynamic and static displacement. The test sample is prepared using a $1.2 \mathrm{~cm} \times 1.2 \mathrm{~cm}$ silicon die evaporated with $2000 \AA$ of $\mathrm{Cr} / \mathrm{Au}$. A $1 \mu \mathrm{m}$ thick layer of Teflon $^{(E)}$ AF $1601 \mathrm{~S}$ is coated on the Au surface and is then implanted with $10 \mathrm{keV}$ electrons using the BLT at $420 \mathrm{mTorr}$ of helium.

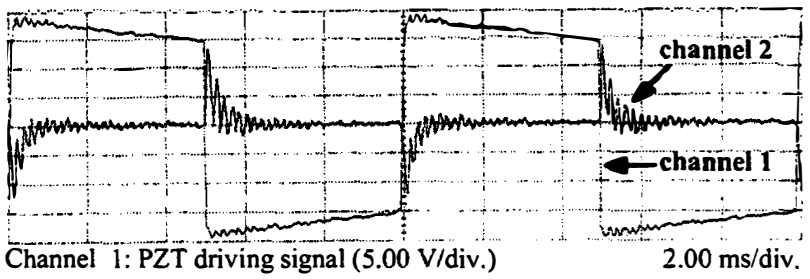

Channel 2: sample generated signal $(50.0 \mathrm{mV} / \mathrm{div}$.)

(a)

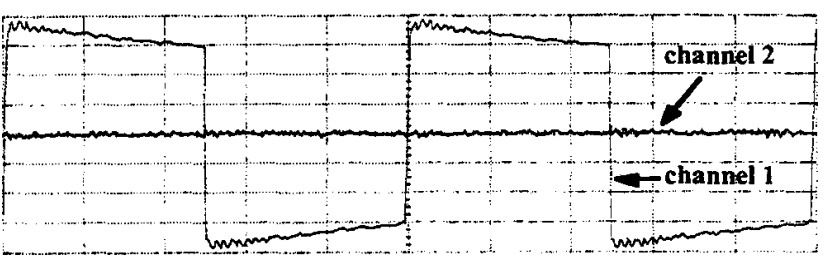

Channel 1: PZT driving signal (5.00 V/div.)

Channel 2: sample signal compensated by $3 \mathrm{~V}(5.00 \mathrm{mV} / \mathrm{div}$.)

(b)

Figure 4. (a) A PZT driving signal and the induced sample signal. (b) The same PZT signal and the compensated sample signal.

The electret sample is fixed on top of the vibrating flexure hinge. The signal generated by induced charges on the stationary electrode due to the vibrating electret is then displayed on the oscilloscope (Figure 3b). By applying a compensation potential, $U_{n}$, between the two electrodes the net electric field in the air gap between the vibrating and stationary electrode can be reduced to zero. The signal generated by the induced charges thus becomes zero. The effective surface charge density, $\rho_{\text {cff }}$, of the electret sample is then given by [5]:

$$
\rho_{\text {eff }}=\varepsilon_{0} \varepsilon U_{0} / t
$$

where $\varepsilon_{0}$ is the permitivity of air, $\varepsilon=1.9$ is the relative permitivity of the Teflon film and $t$ is the electret thickness. Figure 4a shows a typical PZT driving signal at $100 \mathrm{~Hz}$ and the signal generated by the electret sample. Figure $4 \mathrm{~b}$ shows the generated ac signal being compensated to zero by the application of a compensation potential, $U_{\theta}$. Depending on the number of electron pulses, the charge density of an electret sample ranges from $2 \times 10^{-5} \mathrm{C} / \mathrm{m}^{2}$ to $2 \times 10^{-4} \mathrm{C}^{2} \mathrm{~m}^{2}$. The maximum charge density we have obtained is comparable to what has been reported for Teflon films [46].

It is found from experiment that at room temperature the electret initially undergoes a $10-20 \%$ drop in total charge density a few hours after the implantation, but then stabilizes afterward as shown in Figure 5. Some samples were monitored at room temperature over a period of six months (the longest observation time so far up to the point of this paper) and no detectable charge decay was observed. Samples have also been tested for charge decay at elevated temperatures. Curve (a) of Figure 6 shows the charge density of a sample at $100{ }^{\circ} \mathrm{C}$ for 16 hours. The initial significantly large $40 \%$ drop in the charge density is due to the elevated temperature. However, even at $100{ }^{\circ} \mathrm{C}$ the charge stabilizes after the initial drop to a rate which is not measurable within the time span of the experiment. The same electret sample is then monitored for charge decay at $120^{\circ} \mathrm{C}$ as shown by curve (b) of Figure 6. Again there is an initial drop in charge density, but the charge stabilizes after a few hours. The same trend is observed for the same sample at $140^{\circ} \mathrm{C}$, as illustrated by curve (c) in Figure 6 . Although only short term data has been available so far, the 
charge decay data obtained at room and elevated temperatures suggest that a stable electret can be formed using Teflon ${ }^{\circledR} \mathrm{AF}$ and the BLT.

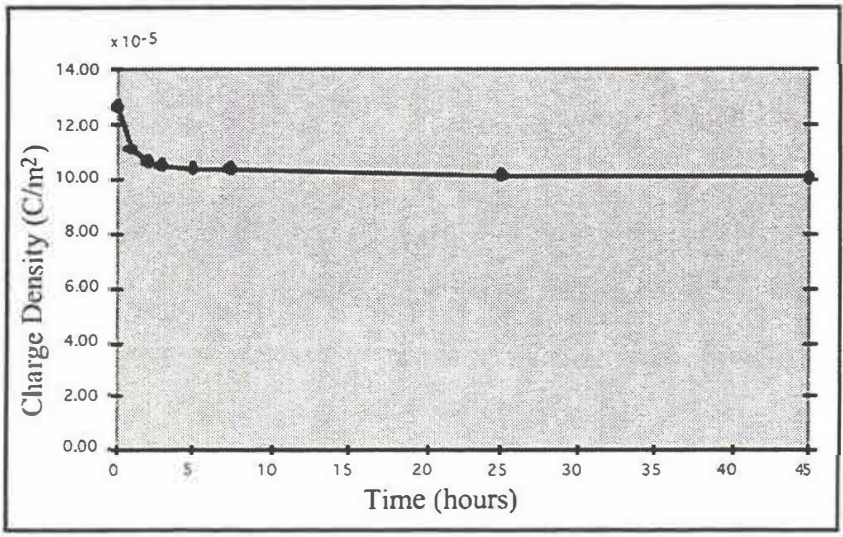

Figure 5. Charge density in Teflon ${ }^{\mathbb{2}} A F$ electret at room temperature.

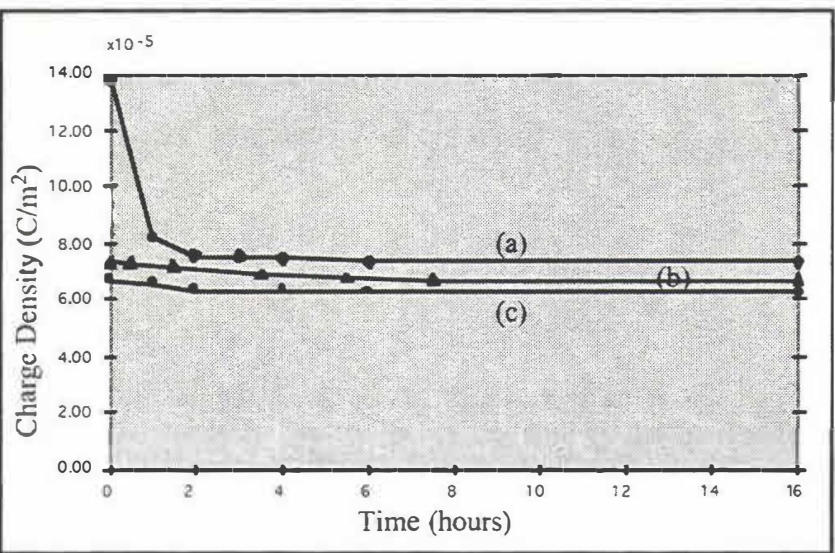

Figure 6. Charge density in Teflon ${ }^{\otimes} A F$ electret at elevated temperatures.

\section{ELECTRET MICROPHONE}

To demonstrate the self-powering capability of a MEMS compatible electret device, a micromachined electret microphone has been fabricated and tested. The fabrication of the membrane part of the microphone begins with a wafer coated with $1 \mu \mathrm{m}$ thick low stress LPCVD nitride. It was first anisotropically back-etched to form a $3.5 \mathrm{~mm} \times 3.5 \mathrm{~mm}$ free standing nitride diaphragm. The front side of the diaphragm was then evaporated with $2000 \AA$ thick $\mathrm{Cr} / \mathrm{Au}$ through a physical mask to form the membrane conductor. The Teflon film was then spun on. Electrons of $10 \mathrm{keV}$ energy were implanted into the Tefion using the BLT. The back plate electrode was fabricated starting with a wafer coated with $3 \mu \mathrm{m}$ thermal oxide. Portions of the oxide layer were etched to create etching windows which extended to the silicon substrate. A timed $\mathrm{KOH}$ etch followed, creating a $3 \mu \mathrm{m}$ deep recess in the silicon substrate. This forms the air gap of the capacitive microphone. A $3 \mu \mathrm{m}$ thick thermal oxide was then grown. A 40 $\times 40$ array of cavities for reducing the air streaming resistance [7] was then formed by anisotropic followed by isotropic etching through the patterned oxide. Each cavity has a $30 \mu \mathrm{m}$ diameter opening and a half-dome shaped hole $80 \mu \mathrm{m}$ in diameter and 50 $\mu \mathrm{m}$ deep. Lastly, a $2000 \AA$ thick $\mathrm{Cr} / \mathrm{Au}$ electrode in the back

plate was evaporated using a physical mask. Figure 7 shows the process flow of the micromachined microphone. The fundamental resonant frequency of the microphone membrane with $\mathrm{Cr} / \mathrm{Au}$ electrode and Teflon film was measured using a laser Doppler vibrometer. The fundamental resonant frequency was found to be around $8 \mathrm{kHz}$. The microphone membrane and back plate are shown in Figure 8a. A portion of the back plate cavity array with conducting gold is shown in Figure $8 \mathrm{~b}$. The hybrid microphone package is illustrated in Figure 9. The two halves of the microphone are mechanically clamped together and are enclosed in a metal box that provides EM shielding.

\section{Microphone Membrane}
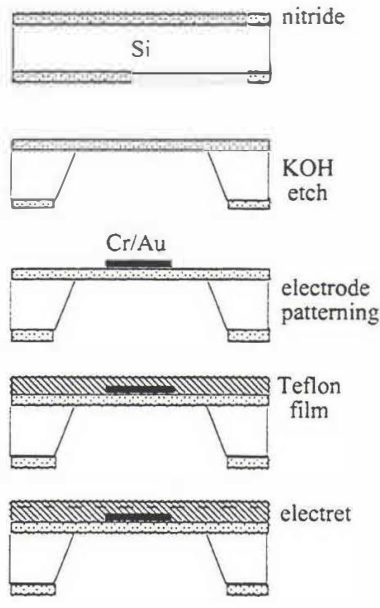

Figure 7. The process flow for the electret microphone.

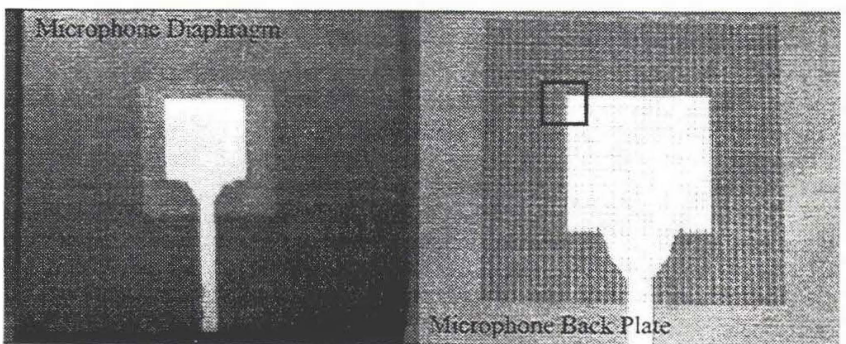

(a)

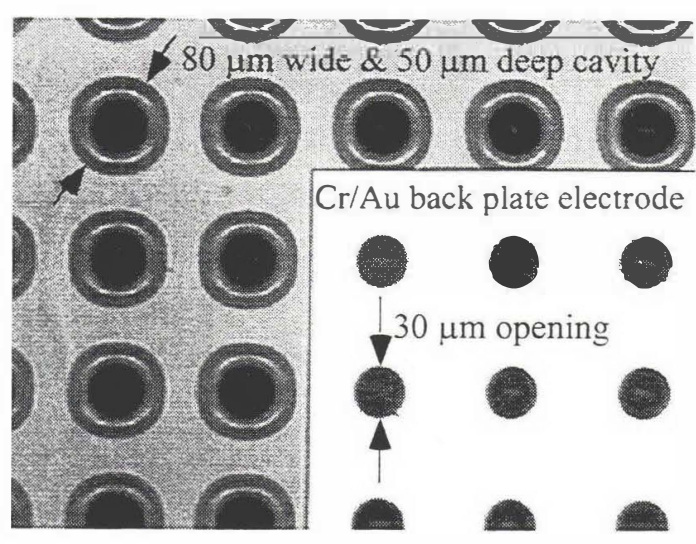

(b)

Figure 8. (a) The microphone diaphragm and back plate. (b) Part of the microphone back plate cavity array with the $\mathrm{Cr} / \mathrm{Au}$ electrode.

\section{Microphone Back Plate}

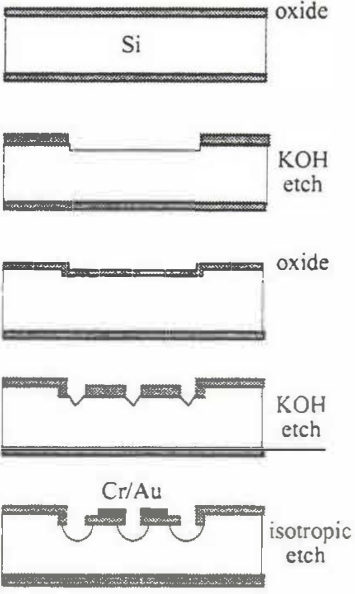

tch 
To reduce the stray capacitance, the electrode area was designed so that it only covered a fraction of the membrane and back plate area [8]. Only a $2 \mathrm{~mm} \times 2 \mathrm{~mm}$ square $\mathrm{Cr} / \mathrm{Au}$ electrode was used to cover the center part of the $3.5 \mathrm{~mm} \times 3.5$ $\mathrm{mm}$ diaphragm and $4 \mathrm{~mm} \times 4 \mathrm{~mm}$ perforated back plate. The fraction of the back plate area occupied by the cavity openings is 0.07 . The streaming resistance, $R_{o}$, is calculated to be 0.03 $\mathrm{Ns} / \mathrm{m}[7,9]$. The cut-off frequency $\left(f_{c}=13.57 \sigma h /\left\{2 \pi R_{a}\right\}\right.$, where $\sigma=100 \mathrm{MPa}$ is the nitride diaphragm stress and $h=1$ $\mu \mathrm{m}$ is the diaphragm thickness [9]) is calculated to be approximately $7.6 \mathrm{kHz}$.

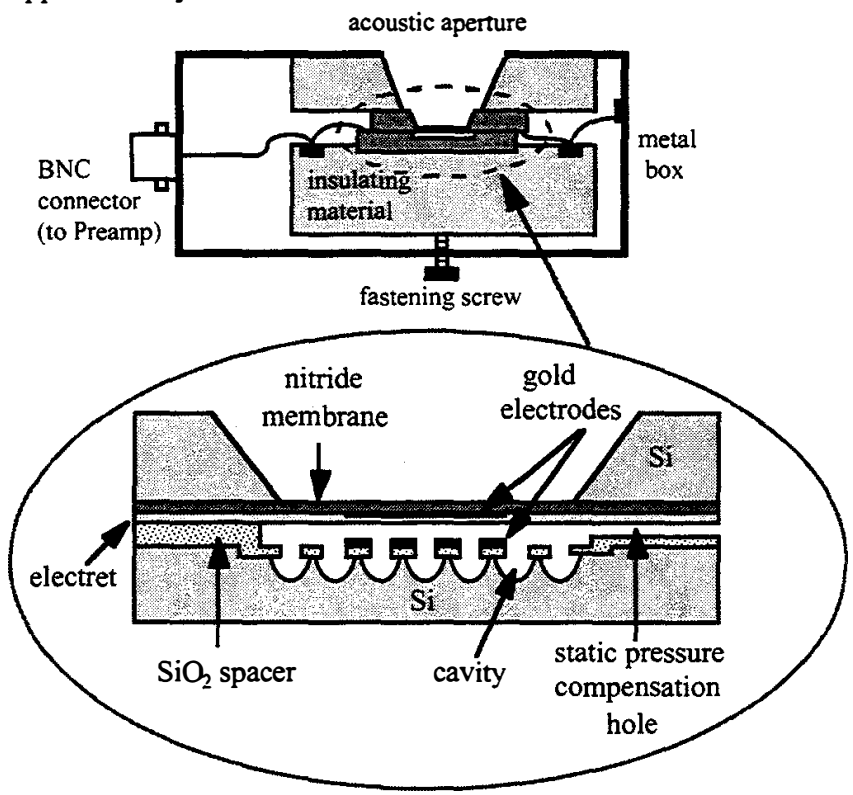

Figure 9. The hybrid microphone package.

The theoretical capacitance of the microphone is $7 \mathrm{pF}$ with the $4.5 \mu \mathrm{m}$ air gap, the $1 \mu \mathrm{m}$ thick Teflon electret and an electrode area of $4 \mathrm{~mm}^{2}$. Using a Hewlett Packard $4192 \mathrm{LF}$ Impedance Analyzer the measured capacitance of the microphone package was $30 \mathrm{pF}$. The discrepancy in capacitance values can be attributed to stray capacitance between the $\mathrm{Cr} / \mathrm{Au}$ electrodes and silicon substrates and between the two clamped halves of the microphone.

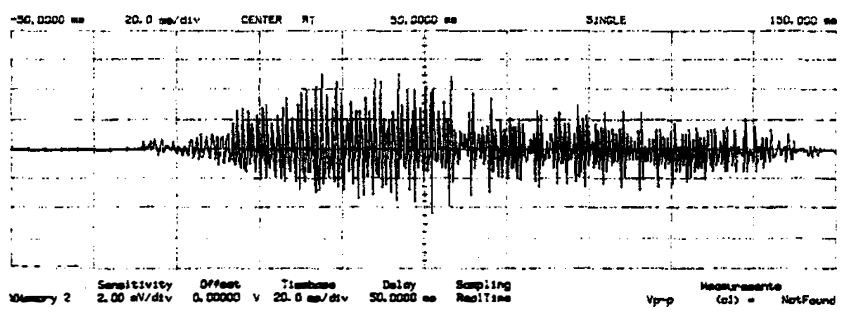

Figure 10. An oscilloscope display of a human voice detected by the hybrid microphone without an amplifier.

The microphone was able to detect the sound from a loud human voice without the use of an amplifier (Figure 10). When the microphone was connected to an EG\&G PARC Model 113 Pre-amp (gain set at 1000) and was excited by a Brüel \& Kjaer Type 4220 Pistonphone operating at $250 \mathrm{~Hz}$ and $123.9 \mathrm{~dB}$ (re. $20 \mu \mathrm{Pa}$ ) amplitude, the oscilloscope displayed a $250 \mathrm{~Hz}, 190$ $\mathrm{mV}$ peak-to-peak amplitude signal. The estimated open-circuit sensitivity of the microphone package is $0.3 \mathrm{mV} / \mathrm{Pa}$. Taking into account the $15 \mathrm{pF}$ input capacitance of the pre-amplifier and the stray capacitance of the microphone package, the open- circuit sensitivity of the microphone itself should be higher than the estimated value. The open-circuit sensitivity of the microphone can also be estimated by calculating the deflection of the electret diaphragm and the output voltage due to a sound pressure [5, 10]. Assuming piston-like movement of the conducting area of the nitride diaphragm, calculations indicate that an open-circuit sensitivity of $7 \mathrm{mV} / \mathrm{Pa}$ is achievable.

\section{CONCLUSIONS}

We have demonstrated the technology to make high charge density, thin film electrets using Du Pont Teflon ${ }^{*}$ AF and the Back Lighted Thyratron. Experiments have been conducted to demonstrate the stability of the Teflon electret at room temperature and at elevated temperatures. A prototype hybrid micromachined electret microphone has been made and preliminary tests and calculations suggest potentially high sensitivities.

\section{ACKNOWLEDGMENT}

This research was funded by the David and Lucile Packard Foundation and by the NSF Presidential Young Investigator Award under NSF Grant No. ECS-9157844.

\section{REFERENCES}

1. G. F. Kirkman and M. A. Gundersen, "A Low Pressure Light Initiated Glow Discharge Switch for High Power Applications", Appl. Phys. Lett. 49, 494 (1986).

2. T-Y Hsu, "A Novel Electron Beam Source Based on the Back-Lighted Thyratron", Ph.D. dissertation, University of Southern California, 1992.

3. T-Y Hsu, K Frank, and M. A. Gundersen, "Collimating Structure for Intense Pseudospark Electron Beams", IEEE International Conference on Plasma Science, New Mexico, June 6-8, 1994

4. B. Hilczer, and J. Malecki, "Electrets", Elsevier Science Publishers, 1986.

5. G. M. Sessler, editor, "Electrets", Springer-Verlag Berlin Heidelberg, 1987.

6. Y. Tada, "Experimental Characteristics of Electret Generator, using Polymer Film Electrets", Japan J. Appl. Phys. 31,846 (1992)

7. Z. Skvor, "On the Acoustical Resistance due to Viscous Losses in the Air Gap of Electrostatic Transducers", Acoustica 19, $295(1967 / 68)$.

8. J. A. Voorthuyzen, A. J. Sprenkels, A. G. H. Van Der Donk, P. R. Scheeper and P. Bergveld, "Optimization of Capacitive Microphone and Pressure Sensor Performance by Capacitorelectrode Shaping", Sensors and Actuator A, 25-27, 331 (1991)

9. P. R. Scheeper, A. G. H. van der Donk, W. Olthuis, and P. Bergveld, "Fabrication of Silicon Condenser Microphones using Single Wafer Technology", J. of Microelectromechanical Systems, vol. 1 (3), 147 (1992).

10. M. G. Allen, M. Mehregany, R. T. Howe, and S. D. Senturia, "Microfabricated Structures for the In Situ Measurement of Residual Stress, Young's Modulus, and Strain of Thin Films", Appl. Phys. Lett. 51 (4), 241 (1987). 